\title{
Pulmonary artery aneurysm
}

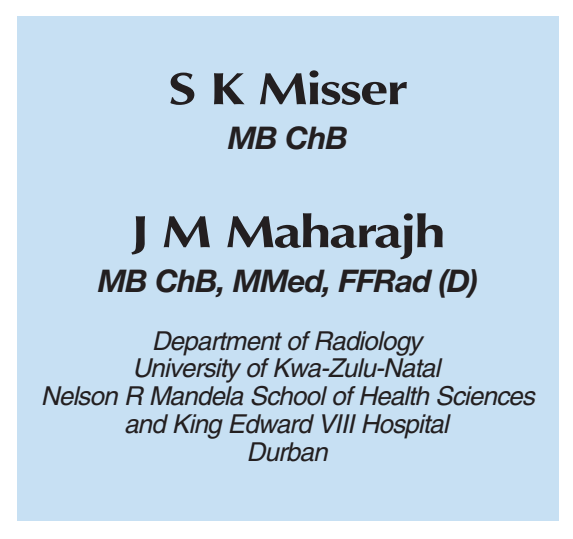

\section{Introduction}

Pulmonary artery aneurysms are a rare finding in general radiological practice. The possible causes are myriad and diverse in pathophysiology. Patients with post-stenotic dilatation of the main pulmonary artery usually present fairly late with insidious cardiorespiratory symptoms. Diagnosis requires radiological imaging assistance as the clinical findings are nonspecific.

\section{Case report}

A 73-year-old woman presented to our medical outpatient department with gradually progressive dyspnoea over the past 2 years. The breathlessness was initially present on exertion. At the time of assessment, however, she had progressed to grade III dyspnoea with associated palpitations, especially on exertion. She had no other significant preceding medical history.

Clinical examination revealed early diastolic and long mid-systolic murmurs along the left heart border.
She also had a $3 \mathrm{~cm}$ hepatomegaly and mild right heart failure. An electrocardiogram (ECG) revealed slight right axis deviation and ventricular extrasystoles.

A chest radiograph done on admission demonstrated massive expansion of the left cardiac border with markedly increased cardiothoracic ratio. There were no radiological features of overt cardiac failure. The aortic outline was clearly delineated with some age-related tortuosity. Considerations at this point included giant left atrium, pulmonary artery aneurysm, giant right atrium, massive pericardial cyst or effusion (Fig. 1).

We then proceeded to perform a computed tomography scan, which revealed a massively dilated main pulmonary artery as well as dilated right and left pulmonary arteries. Slight

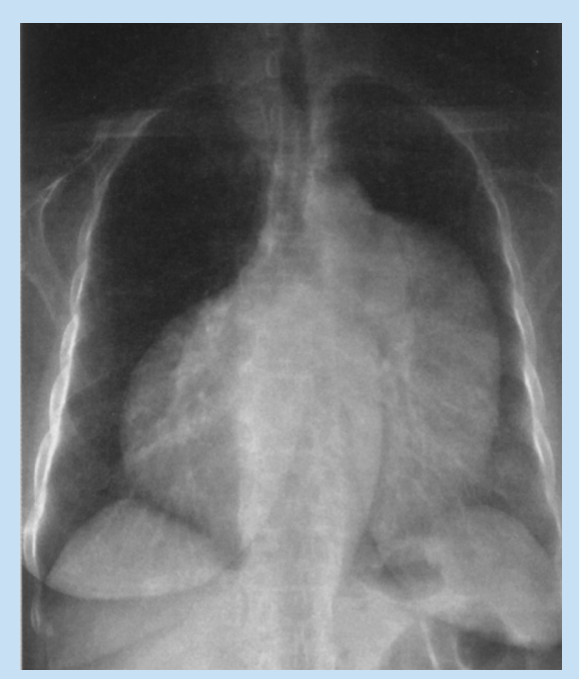

Fig. 1. Postero-anterior chest radiograph performed on admission. right atrial and right ventricular dilatation was also noted. The rest of the mediastinal structures were within normal limits. A diagnosis of aneurysm of the main pulmonary artery was made (Fig. 2).

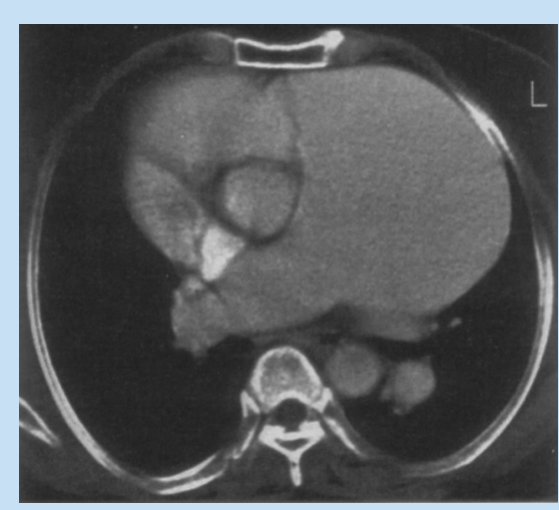

Fig. 2. Axial CT scan of the chest at the level of the main pulmonary artery.

An echocardiogram confirmed pulmonary valvular stenosis with post-stenotic dilatation and pulmonary artery aneurysm formation. The pulmonary valve pressure gradient was $>28 \mathrm{mmHg}$. The patient settled on low-dose diuretic therapy, and following cardiothoracic surgical consultation it was decided that no surgical intervention was warranted. She was followed up at the clinic.

\section{Discussion}

Aneurysms of the pulmonary artery (PAA) are rare in adults. Predisposing conditions include congenital and acquired heart disease, e.g. left-to-right shunting or pulmonary valve stenosis with post-stenotic dilatation. Other causes include infection (tuberculosis, syphilis, osteomyelitis, pneumonia), systemic vasculitides (Hughes-Stovin's disease, Behcet's disease), collagen vascular diseases, connective tissue disorders, (Marfan's syndrome, Ehler's-Danlos 
syndrome), trauma (direct or blunt chest injury), mucoid vasculopathic changes and idiopathic PAA. ${ }^{1}$

Irrespective of the underlying cause, most PAA are associated with pulmonary arterial hypertension. Clinically patients usually present with symptoms of mild congestive heart failure of insidious onset, haemoptysis and bronchial compression, or the aneurysm is discovered incidentally on chest radiography for unexplained dyspnoea. ${ }^{2}$

Post-stenotic dilatation of the pulmonary artery is a common feature of pulmonary stenosis, but does not usually reach aneurysmal proportions. In most cases of post-stenotic dilatation the main or left pulmonary artery is involved. One plausible explanation for this is the sharp angle at which the right pulmonary artery emerges, causing the near-vertical, high-velocity jet to bypass its origin. ${ }^{3}$ In the patient reported here the degree of dilatation was marked and both right and left pulmonary arteries were involved. A possible associated degenerative process (especially since she was in her 8th decade) is thought to be responsible for this appearance.

Clinical response to routine antifailure/diuretic therapy is usually good, but the response cannot be predicted. Long-term outcome of untreated PAA is not well documented. One-third of patients with PAA, from all causes, will die from aneurysm rupture. Aneurysms associated with post-stenotic dilatation persist after treatment but are of no clinical significance. Recent reports have described the use of balloon valvuloplasty in correcting the valvular stenosis with resultant reduction in pulmonary arterial pressures and aneurysm stability. ${ }^{4}$

Our patient responded very well to medical therapy and her symptoms have almost completely settled. We shall continue to re-assess her clinical status as well as the trans-pulmonary valve pressure gradient as an index of stability.

\section{Acknowledgements}

Special thanks to Professor P Corr for editing the manuscript, $\mathrm{Mr}$ Steve Odayan (Chief Radiographer) for assistance with imaging and Ms Marianne Singh for technical support.

\section{References}

1. Deterling RA, Clagett OT. Aneurysms of the pulmonary artery. Am Heart J 1947; 34: 471.

2. Lopez-Candales A, Kleiger RE, Aleman-Gomez J, Kouchoukos NT, Botney MD. Pulmonary artery aneurysm: Review and case report. Clin Cardiol 1995; 18: 738-740.

3. Tami LF, McElderry MW. Pulmonary artery aneurysm due to severe congenital pulmonic stenosis. Case report and literature review. Angiology 1994; 45: 383-390.

4. Butto F, Lucas RV, Edwards JE. Pulmonary artery aneurysm: a pathological study of five cases. Chest 1987; 91: 237-240. 